\title{
Towards Location-Based Personalized Voice Web Search on Smartphones
}

\author{
Dae Young Choi* \\ Dept. of MIS, Yuhan Univ., Puchon City, South Korea. \\ * Corresponding author. Tel: +82-2-2610-0838; email: dychoi@yuhan.ac.kr \\ Manuscript submitted June 5, 2015; accepted September 8, 2015. \\ doi: $10.17706 /$ jcp.11.1.62-71
}

\begin{abstract}
Web search engines used in smartphones need to be refined to capture the user's perception and user's search intentions. However, to design such a search engine is not trivial. Fuzzy query may be considered to obtain the user's perception and user's search intentions in voice Web search on smartphones. Handling fuzzy query in voice Web search on smartphones is one of the most difficult problems. It is mainly derived from the complexity and the degree of freedom of natural language. To reduce the complexity and the degree of freedom of fuzzy query in voice Web search on smartphones, attribute-driven approach for fuzzy query is proposed. In this paper, we design a prototype model for handling fuzzy query in voice Web search on smartphones. It is based on voice Web search, user's physical location, search distance, search directory and user's search intentions. The filtering ratio on search results of the proposed approach compared to existing smartphones is presented. In addition, by using the values of attributes for fuzzy term(s), we can achieve perception personalization and Web search engines return the personalized search results that users really want. It may be considered as a new explicit personalization method based on the values of attributes for fuzzy term(s) in smartphones. The proposed method is a further step towards location-based personalized voice Web search (LPVWS) for smartphone users.
\end{abstract}

Key words: Fuzzy query, perception personalization, location-based personalized voice web search (lpvws), user's search intentions.

\section{Introduction}

There is a rising public demand for voice-enabled smartphone applications, because it is the safest and most natural interface of all [1], [2]. Now, Google provides smartphone users with voice search [3]. For example, avoid typing your search query while searching for nearby businesses (e.g. 'gas station', 'pizza', 'sushi'). A smartphone user can download voice search application from Google Mobile App [3]. Using the voice search application in Google phone, a user speaks search queries in English, Japanese, etc. Similarly, iPhone makes a phone call, plays music, and accomplishes even more using only your voice [4]. Spoken queries are natural medium for searching the Web. A speech interface to the Google search engine is described and experiments with various statistical language models of their prototype system is presented in [5]. However, there is a lot of room for improvement in the recall rate.

In general, existing smartphones make voice Web search by using keywords and user's current location. However, existing smartphones have some problems in handling fuzzy query in voice Web search. Voice Web search on smartphones is one of the most difficult problems. It is mainly derived from the complexity and the degree of freedom of natural language. To reduce the complexity and the degree of freedom of fuzzy query in 
voice Web search on smartphones, a prototype model for handling fuzzy query in voice Web search on smartphones is proposed. It is based on voice Web search, user's physical location, search distance, search directory and user's search intentions.

Increased capabilities of computer hardware and software have created a vast body of machine-readable resources. However, smartphones users in existing voice Web search, more often, seeking needles in haystacks, are overwhelmed by the quantity of irrelevant information. Often this is caused by a poor query (too vague or too generic; for example, try searching for "cars"). Without the context of the query and the relations of the information, Web search engines in smartphones are doomed to return random samples of the Internet. Despite numerous refinements, most voice Web search engines on smartphones still return too many results and random samples of the Internet. In other words, they often give users a bunch of garbage. In this respect, we need a new tool to handle the removal of spurious returns in voice Web search on smartphones. To make targeting Web search results in voice Web search on smartphones, a prototype model for handling fuzzy query is proposed. It provides smartphone users with location-based personalized voice Web search (LPVWS).

The rest of the paper is organized as follows: A prototype model for handling fuzzy query in voice Web search on smartphones is proposed in Section 2. We introduce a different way of personalization method for smartphones in Section 3. It is the perception personalization based on the values of attributes for fuzzy terms. Three classes of fuzzy terms for representing linguistic quantifiers that cover most used in natural language are suggested and their properties are analyzed in Section 4. We briefly compare the proposed smartphone with existing smartphones, and experimental results are described with filtering ratio $(\Omega)$ on search results in Section 5. We conclude the paper in Section 6.

\section{A Prototype Model}

\subsection{General Mechanism on Search Engines}

A search engine, on receiving a query, would compare the query with its document index (DI) [6]. The DI is generally consisted of keywords. Based on the DI, commercial Web search engines help users to retrieve information. At present, location tag and keywords provide smartphone users with local angle to make searching for local businesses on the Web. However, it is still within the keyword-based local Web search. Although commercial Web search engines such as Yahoo, Google help Internet users get rich information, they generally return too many Web pages irrelevant to the user's query. In addition, they do not properly handle fuzzy query [1], [2], [7], [8].

Voice-enabled smartphone applications are one of promising areas, because it is the safest and most natural interface of all. In general, existing smartphones make voice Web search by using keywords and user's current location. However, they make too much search results generally. In this respect, we need to filter the search result for smartphone users. As a new way of filtering search results on smartphones, we consider the fuzzy query to reflect user's search intentions. Existing smartphones still do not properly process fuzzy queries. For example, they do not properly handle a fuzzy query that finds 'famous sushi' restaurants from user's physical location within a specified area. A specified area is a search distance for Web searching. In this case, 'famous' and 'sushi' are generally processed as independent keywords in current smartphones. As a result, they return many Web pages (or URLs) irrelevant to user's query. It should be noted that the fuzzy term 'famous' is a constraint on the focal keyword 'sushi' rather than an independent keyword. We note that the fuzzy term 'famous' plays the role of a constraint on the fuzzy query. Thus, using fuzzy term(s), smartphone users can narrow thousands of hits to the few that users really want. In this respect, the fuzzy terms in a query provide helpful hints for targeting queries that users really want. However, current smartphones tend to ignore the importance of fuzzy terms in a query. In this fuzzy query, 
we note that the user wants to find 'famous sushi' restaurants, not just 'sushi' restaurants. However, handling fuzzy query in voice Web search on smartphones is one of the most difficult problems. To reduce the complexity and the degree of freedom of fuzzy query in voice Web search on smartphones, a prototype model for handling fuzzy query in voice Web search on smartphones is proposed.

\subsection{Proposed Search Mechanism}

For example, if a traveler is visiting San Francisco and tries to find 'famous sushi' restaurants within 2 miles from a smartphone user on the Web, he/she tells a fuzzy query 'famous sushi'. In this case, the fuzzy term 'famous' is a constraint on the focal keyword 'sushi' and the fuzzy term is processed as follows :

STEP 1: A smartphone user tells the fuzzy query 'famous sushi' to smartphone. Then the smartphone saves the user's voice signal information in a temporary storage.

STEP 2: Display search directories such as restaurants, entertainments, etc. Then user selects the directory that the user wants. It is consisted of attributes related to the directory. For example, in case of the directory 'restaurants', a kind of 'restaurants' (Japanese, Chinese, etc.), prices of foods and search distance may be suggested as attributes.

STEP 3: To handle the fuzzy term 'famous' related to the focal keyword 'sushi', the attributes such as 'visitors/day', 'tastiness' may be considered. To reflect user's preferences, a user selects the values of attributes.

STEP 4: By using search directory, focal keyword, search distance and attributes for handling the fuzzy term 'famous' in STEP 1 3 (see Fig. 1), it makes location-based personalized voice Web search on smartphones.

The primary source of imprecision in fuzzy query is fuzziness of class boundaries. To reduce the complexity and the degree of freedom of fuzzy query processing on smartphones, the directory in STEP 2 is used to confine the search domain. In addition, values of attributes for fuzzy term in STEP 3 are used to reflect user's preferences. Thus, the STEP 2 and 3 in proposed prototype model can be used to capture user's search intentions.

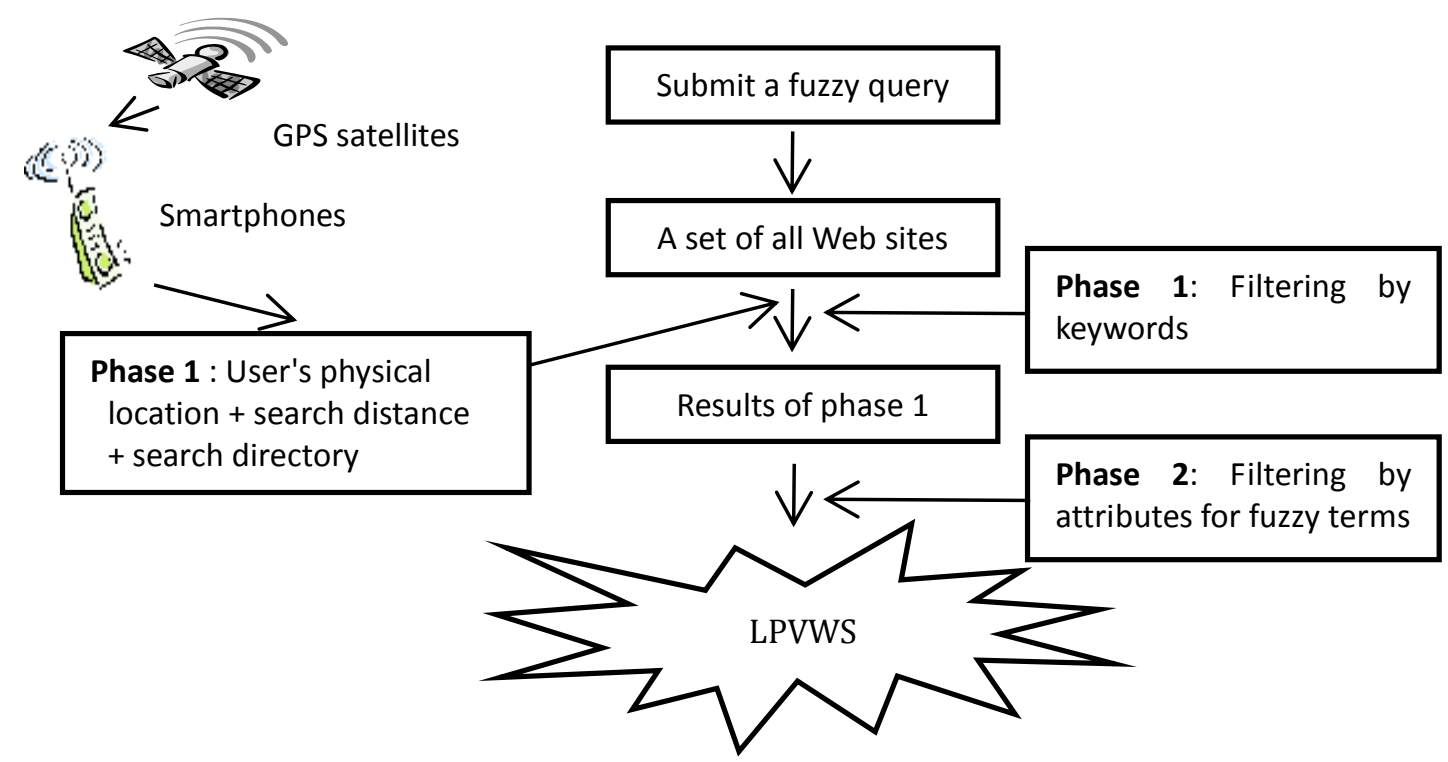

Fig. 1. Location-based personalized voice Web search (LPVWS) on smartphones.

\subsection{Examples}

Let the set of 'sushi' restaurants from user's physical location within a specified area be $A=\left\{A_{1}, A_{2}, \ldots, A_{99}\right.$, 
$\left.A_{100}\right\}$ and each $\mathrm{A}_{\mathrm{i}}$ has its own URL.

EXAMPLE 1: Consider a crisp query that finds 'sushi' restaurants from user's physical location within 2 miles (Q1). In this case, the phase 2 (i.e., attributes for fuzzy terms) in Fig. 1 is not used. Thus, search results are as in Table 1.

Table 1. Snapshot after Processing $Q_{1}$

\begin{tabular}{ccc}
\hline \hline Result of Phase 1 & Attributes & Result of Phase 2 \\
\hline$\left\{A_{1}, A_{2}, \ldots, A_{100}\right\}+$ Irrelevant URLs & - & $\left\{A_{1}, A_{2}, \ldots, A_{100}\right\}+$ Irrelevant URLs \\
& & \\
\hline \hline
\end{tabular}

We note that search results after both phases 1 and 2 in Fig.1 are equal. Thus, users receive locally targeted search results based on the focal keyword 'sushi', user's physical location, search distance and search directory 'restaurants'.

EXAMPLE 2: Consider a fuzzy query that finds 'famous sushi' restaurants from user's physical location within 2 miles $\left(\mathrm{Q}_{2}\right)$. In this case, both phases 1 and 2 in Fig. 1 are used. For handling the fuzzy query 'famous sushi' restaurants, the following information may be used: keyword $=\{$ sushi $\}$, attribute $=\{$ no. of visitors/day\}. We note that a focal keyword 'sushi' is restricted by a fuzzy term 'famous'. In this case, the fuzzy term 'famous' may be manipulated by the number of visitors per day, and represented by a membership function as in Fig. 2. The value of focal attribute 'no. of visitor' (e.g., 'no. of visitors' per day $\geq$ 100 ) is given by a user. We assume that search results of $Q_{2}$ is $A_{F}, A_{F} \in\left\{A_{1}, A_{2}, \ldots, A_{99}, A_{100}\right\}$, by using $\alpha$-cut as in Fig. 2. Thus, smartphone users receive more personalized and locally targeted search results $\left(A_{F}\right)$ based on the focal keyword 'sushi', the value of focal attribute 'no. of visitor', user's physical location, search distance and search directory 'restaurants'.

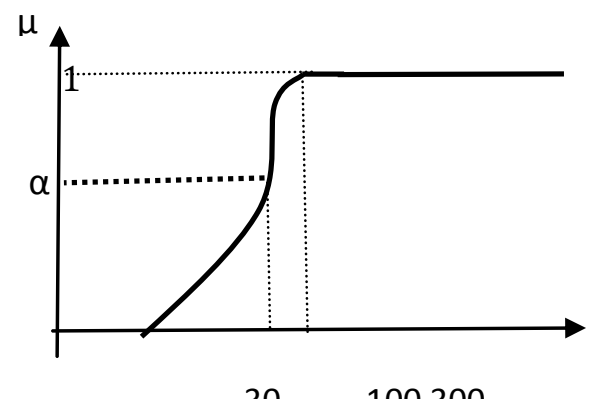

Fig. 2. A membership function of 'famous'.

In this case, search results are in Table 2.

Table 2. Snapshot After Processing $Q_{2}$

\begin{tabular}{ccc}
\hline \hline Result of Phase 1 & Attributes & Result of Phase 2 \\
\hline$\left\{A_{1}, A_{2}, \ldots, A_{100}\right\}+$ Irrelevant URLs & No. of visitors & URLs w.r.t $\left\{\mathrm{AF}_{\mathrm{F}}\right\}$ \\
\hline \hline & \\
\hline
\end{tabular}

The expressive power of voice Web search on smartphones is relatively weak when restricted to keyword-based search. At present, commercial smartphones based on the Document index (DI) present limitations in modeling perceptual aspects of humans. In addition, they generally return a bunch of Web 
pages (or URLs) irrelevant to user's query. Existing smartphones do not properly handle the fuzzy terms representing human's perception. In addition, they appear to have trouble with returning the targeted results.

Many Web search in existing smartphones support Boolean operators, field searching, and other advanced techniques such as fuzzy logic in a very primitive way. While searches may retrieve thousands of hits, finding relevant partial matches and query relevant information with deductive capabilities might be a problem. What is also important to mention for search engines is query-relevant information rather than generic information. Therefore, the Web search engines need to be refined to capture the user's perception and user's search intentions. However, to design such a search engine is not trivial. Q/A(Question/Answer) systems can be used as a first step to build a knowledge base for search engines or to capture some of user's perceptions and user's search intentions [9]. To capture some of user's perceptions and user's search intentions, new machineries and tools need to be developed. Therefore, we envision that non-classical techniques such as fuzzy logic based-clustering methodology, fuzzy similarity, fuzzy aggregation, etc., are required. The STEP 2 and 3 in the proposed prototype model might be used to capture some of user's perceptions and user's search intentions. They are regarded as new Q/A components for handling fuzzy query in voice Web search on smartphones.

\section{Personalization in Web Search Results}

Personalization technology gives the user a more tailored site [10]. On a Web site, personalization is the process of tailoring pages to individual users' characteristics or preferences [1], [2], [7], [8]. Commonly used to enhance customer service or e-commerce sales, personalization is sometimes referred to as one-to-one marketting, because the enterprise's Web page is tailored to specifically target each individual consumer. Personalization is a means of meeting the customer's needs more effectively and efficiently, making interactions faster and easier and, consequently, increasing customer satisfaction and the likelihood of repeat visits. Personalization in some ways harkens back to an earlier day, by making consumer relationships more closely tailored to the individual. If you've ever bought a book from Amazon, for example, the next time you visit they will - like a friendly and helpful sales clerk - greet you by name and tell you about products in stock that they think you might like (such as more books by the same author, or books purchased by other people who also bought the book that you purchased). Many portal sites, such as Yahoo allow site visitors to customize the page with selected news categories, local weather reports, and other features [11].

The Web is an impressive success story in terms of both its available information and the growth rate of human users. It now penetrates most areas of our lives, and its success is based on its simplicity. Unfortunately, this simplicity could hamper further Web development [7], [12]. Often, search engines will return many results, but most will be uninteresting to the user. If a search tool can get geographical information or learn preferences or contexts from the user, this information can be used to improve the search or document-ranking process. Web pages are personalized based on the characteristics (interests, social category, context, etc.) of an individual. Personalization are generally based on implicit data, such as items purchased or pages viewed. There are three broad methods of personalization:

- Implicit

- Explicit

- Hybrid

With implicit personalization the personalization is performed by the Web page. With explicit personalization, the Web page is generally changed by using the features provided by user. Hybrid personalization combines the above two approaches to leverage the best of both. Many companies offer 
services for Web recommendation and email recommendation that are based on personalization or anonymously collected user behaviors.

Internet users do not have to watch all the irrelevant noise. In this respect, personalization technology gives the user a more tailored site. It is the provision to the individual of tailored products, services, advertisements, or information relating to products or services. Personalization of Web pages can be accomplished in numerous ways. Some approaches require the user's participation (typically through filling out a form or questionnaire). Other approaches operate behind the scenes, without depending on user input, for example, by using Web cookies for tracking what user likes to view or by analyzing Web server log for the access pattern and statistics, etc. Search engine logs provide a wealth of information that machine learning techniques can harness to improve search quality. A community-based personalizing Web search approach can leverage the latent knowledge created with in communities by recording users' search activities (i.e., the queries they submit and results they select) at the community level. They can use this data to build a relevance model that guides the promotion of community-relevant results [13].

Google's Web history uses more detail private search activity [14]. Web history can tell you about these and other interesting trends in your Web activity. An important problem relating to personalization concerns understanding how a machine can help an individual user via suggesting recommendations [15]. In this respect, we mainly present the perception personalization based on the values of attributes for fuzzy query in voice Web search on smartphones. In general, different people may use different conceptual comprehension (fuzzy terms, membership functions, $\alpha$-cut), with respect to the same situation. Thus, by using the values of attributes for fuzzy terms, we can achieve perception personalization and Web search engines return the personalized search results that users really want. It may be considered as a new explicit personalization method based on the values of attributes for fuzzy terms in smartphones. It may be also considered as a solution relating to personalization for 'understanding how a machine can help an individual user via suggesting recommendations' described in [15].

In this paper, we introduce a different way of personalization method for smartphones. It is the perception personalization based on the values of attributes for fuzzy terms. Together with existing personalization methods, the proposed perception personalization may be used as a complementary and synergistic rather than competitive.

\section{Classification of Fuzzy Terms}

Zadeh [16] suggested we can represent linguistic quantifiers as fuzzy subsets of the unit interval. In this representation the membership grade of any proportion $r \in[0,1], Q(r)$, is a measure of the compatibility of the proportion $r$ with the linguistic quantifier we are representing by the fuzzy subset $Q$. For example, if $Q$ is the quantifier 'most' then $Q(0.9)$ represents the degree to which 0.9 satisfies the concept 'most'. Yager [17] identified three classes of linguistic quantifiers that cover most of these used in natural language.

1) A quantifier $Q$ is said to be monotonically nondecreasing if $r 1>r 2$ then $Q(r 1) \geq Q(r 2)$.

2) A quantifier $Q$ is said to be monotonically nonincreasing if $r 1>r 2$ then $Q(r 1) \leq Q(r 2)$.

3) A quantifier $Q$ is said to be unimodal if there exists two values $a \leq b$ both contained in the unit interval such that for $r<a, Q$ is monotonically nondecreasing, for $r>b, Q$ is monotonically nonincreasing, and for $r \in[a, b], Q(r)=1$.

Similarly, we can identify three classes of fuzzy terms that cover most used in natural language. For example, we may consider the fuzzy term 'famous' as monotonically nondecreasing case as shown in Fig. 2.

EXAMPLE 3: Consider a fuzzy query that finds 'low-price sushi' restaurants from user's physical location within 2 miles $\left(Q_{3}\right)$. In this case, the fuzzy term 'low-price' is represented as monotonically nonincreasing. 


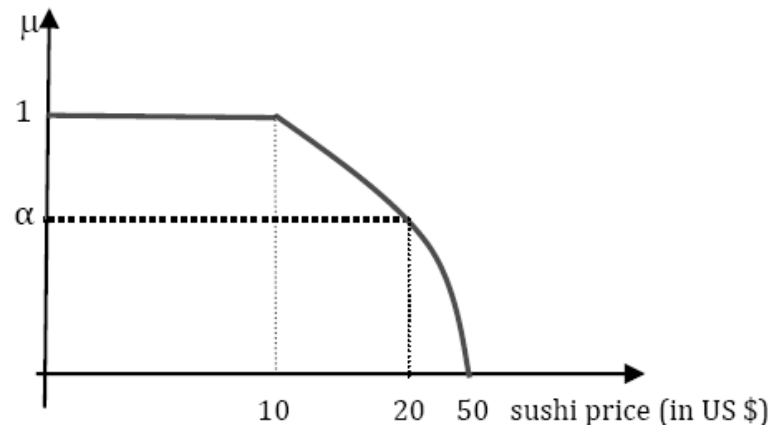

Fig. 3. A membership function of 'low-price'.

EXAMPLE 4: Consider a fuzzy query that finds 'moderate-price sushi' restaurants from user's physical location within 2 miles $\left(Q_{4}\right)$. In this case, the fuzzy term 'moderate-price' is represented as unimodal.

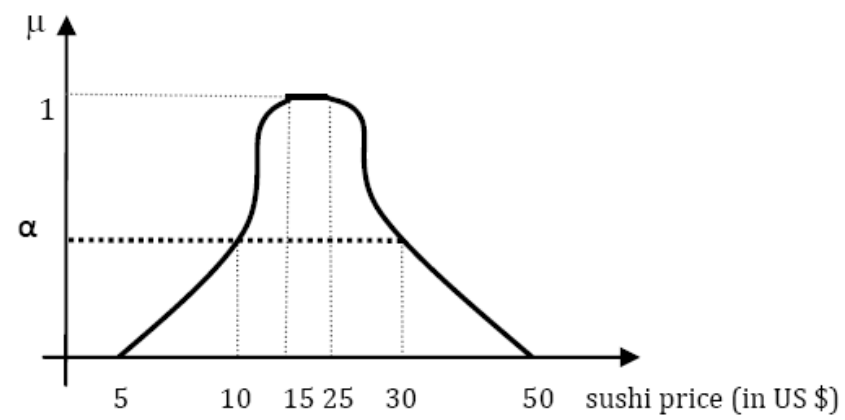

Fig. 4. A membership function of 'moderate price'.

\section{Experiments and Comparisons}

The proposed search mechanism in Fig. 1 can be explained by SQL-like language: SELECT * FROM \{a set of Web sites that satisfies keyword(s), user's physical location, search distance and search directory] [WHERE the value(s) of focal attribute(s) in the directory are satisfied by the user]. We note that existing Web search engines in smartphones tend to ignore the importance of [WHERE] part. The proposed mechanism gives users a more personalized and locally targeted search results in voice Web search on smartphones. We note that user's search intentions can be explicitly reflected by using the values of focal attributes for fuzzy terms in the directory.

Compared to current smartphones, an advantage of the proposed approach is obtained by using the filtering ratio as follows:

Let $\Delta$ be a set of Web sites that satisfies keyword(s) in the Document Index (DI), user's physical location and search distance used in the current smartphones. And let $\delta$ be a set of Web sites that satisfies both keyword(s), user's physical location, search distance and the value(s) of focal attribute(s) for the fuzzy terms, then the effectiveness of the proposed approach compared to the current smartphones becomes $\delta / \Delta$, where $0 \leq \delta / \Delta \leq 1$. We note that if we get a smaller $\delta / \Delta$, then more personalized search results (i.e., more reduced search results) are obtained. Let $\Omega$ be the filtering ratio on search results of the proposed approach compared to the current smartphones, then $\Omega$ is computed as $\Omega=(1-(\delta / \Delta)) \times 100$. To carry out experiments on the filtering ratio $(\Omega)$ on search results of the proposed approach compared to the current smartphones, we consider three classes of fuzzy terms in Section 3.

1) Monotonically nondecreasing case

We use test file with 100 records. Each record $i(i=1,2, \ldots, 100)$ has $<$ keyword $_{i}$ attribute_value $_{i}>$. That is, 100 records are consisted of 100 keywords (i.e., restaurant names) for 'famous sushi' restaurants and 100 attribute values (e.g., 'no. of visitors' per day in Fig. 2) for handling the fuzzy term 'famous'. In this case, the 
filtering ratio $(\Omega)$ on search results of the proposed approach compared to current smartphones becomes higher as the values of the attribute increase.

Table 3. A Monotonically Nondecreasing Case

\begin{tabular}{cccccc}
\hline \hline Attribute Values & 0 & 50 & 100 & 200 & 300 \\
\hline$\delta$ & 100 & 75 & 53 & 21 & 3 \\
\hline$\Delta$ & 100 & 100 & 100 & 100 & 100 \\
\hline$\Omega$ & $0 \%$ & $25 \%$ & $47 \%$ & $79 \%$ & $97 \%$ \\
\hline \hline
\end{tabular}

In a monotonically nondecreasing case, as the values of the attribute (e.g., 'no. of visitors' per day) increase, a higher filtering $\operatorname{ratio}(\Omega)$ is obtained (i.e., more reduced search results).

2) Monotonically nonincreasing case

We use test file with 100 records. In this case, 100 keywords (i.e., restaurant names) for 'low-price sushi' restaurants and 100 attribute values (e.g., Sushi price in Fig. 3) for handling the fuzzy term 'low-price' are used. In this case, the filtering $\operatorname{ratio}(\Omega)$ on search results of the proposed approach compared to current smartphones becomes lower as the values of the attribute increase.

Table 4. A Monotonically Nonincreasing Case

\begin{tabular}{cccccc}
\hline Attribute Values & 5 & 10 & 20 & 40 & 50 \\
\hline$\delta$ & 3 & 25 & 54 & 78 & 100 \\
\hline$\Delta$ & 100 & 100 & 100 & 100 & 100 \\
\hline$\Omega$ & $97 \%$ & $75 \%$ & $46 \%$ & $22 \%$ & $0 \%$ \\
\hline
\end{tabular}

In a monotonically nonincreasing case, as the values of the attribute (e.g., Sushi price) increase, a lower filtering ratio $(\Omega)$ is obtained (i.e., less reduced search results).

3) Unimodal case

We use test file with 100 records. In this case, 100 keywords (i.e., restaurant names) for 'moderate-price sushi' restaurants and 100 attribute values (e.g., Sushi price in Fig. 4) for handling the fuzzy term 'moderate-price' are used. In this case, the filtering ratio $(\Omega)$ on search results of the proposed approach compared to current smartphones becomes higher as the values of the attribute come closer to $\beta$ ( $\beta$ denote the midpoint between between $a_{i}$ and $b_{i}$ derived from $\alpha$-cut $\left[a_{i}, b_{i}\right]$. In Fig. $4, \beta$ becomes 20 from $\alpha$-cut [10, 30]).

Table 5. A Unimodal Case

\begin{tabular}{cccccc}
\hline Attribute Values & 5 & 10 & 20 & 30 & 50 \\
\hline$\delta$ & 95 & 57 & 8 & 55 & 97 \\
\hline$\Delta$ & 100 & 100 & 100 & 100 & 100 \\
\hline$\Omega$ & $5 \%$ & $43 \%$ & $92 \%$ & $45 \%$ & $3 \%$ \\
\hline
\end{tabular}

In a unimodal case, as the values of the attribute (e.g., Sushi price) come closer to the midpoint $\beta$, a higher filtering $\operatorname{ratio}(\Omega)$ is obtained (i.e., more reduced search results).

In summary, the filtering $\operatorname{ratio}(\Omega)$ on search results depends on three classes of fuzzy terms (i.e., monotonically nondecreasing, monotonically nonincreasing, unimodal) used in natural language as follows :

1) Monotonically nondecreasing case

The larger the value of focal attribute, the higher the filtering ratio $(\Omega)$ for a given fuzzy query.

2) Monotonically nonincreasing case

The larger the value of focal attribute, the lower the filtering ratio $(\Omega)$ for a given fuzzy query.

3) Unimodal case

If an interval of focal attribute determined by $\alpha$-cut is $\left[a_{i}, b_{i}\right]$, and let $\beta$ denote the midpoint between $a_{i}$ and $b_{i}$, then the degree of closeness to the midpoint $\beta$ can be used as the filtering ratio criterion. That is, the closer the $\beta$, the higher the filtering $\operatorname{ratio}(\Omega)$ for a given fuzzy query. 
We note that experimental results on the filtering ratio $(\Omega)$ in Tables 3-5 are partially affected by misspelling queries, out-of vocabulary words of fuzzy terms. We briefly summarize the differences between the proposed smartphone and existing smartphones in Table 6.

Table 6. Comparisons

\begin{tabular}{lll}
\hline & Proposed Smartphone & Existing Smartphones \\
\hline Handling fuzzy terms & Yes & Limited \\
\hline Search method & Keyword+Location+Fuzzy Terms & Keyword+Location \\
\hline Personalization & Values of attributes & Web cookies, Web server log, etc. \\
\hline- - For the better personalization, both personalization methods are complementary rather than competitive \\
\hline
\end{tabular}

\section{Conclusion}

To reduce the complexity and the degree of freedom of fuzzy query in smartphone, a prototype model for handling fuzzy query in voice Web search on smartphones is proposed. It is based on voice Web search, user's physical location, search distance, search directory and user's search intentions. Experiments on the filtering ratio $(\Omega)$ compared to the current smartphones are carried out. Experimental results show that the filtering ratio $(\Omega)$ depends on three classes of fuzzy terms as in Section 5.

In this paper, we also introduce a different way of personalization method for smartphones. It is the perception personalization based on the values of attributes for fuzzy terms. For the better personalization, existing personalization methods and proposed perception personalization may be used as a complementary and synergistic rather than competitive.

Recently, Google is continuously trying to find intelligent search methods for reflecting user's search intentions on Web searching. Google launches knowledge graph, a new intelligent search platform [18]. Google's goal is to get you to the information you're looking for in fewer clicks, while also increasing the relevancy to user's search intentions. Google's calling this the first 'baby step' towards a 'Star Trek Computer' future [18]. As a new way for reflecting user's search intentions, the proposed approach could be also applied. The proposed approach provides smartphone users with more personalized and locally targeted search results. It is a further step towards location-based personalized voice Web search (LPVWS) for smartphone users.

Future works may be extended in several directions based on the current proposal: First, to handle various fuzzy queries, classifying directories and analyzing their attributes could be developed. Second, there is a need for some field works to test the value of the proposed approach. Third, handling multiple fuzzy terms in a fuzzy query could be developed. For example, a fuzzy query that finds 'famous and low-price sushi' restaurants. In this case, some weighting methods for handling multiple fuzzy terms could be developed.

\section{Acknowledgment}

The author wish to thank prof. L. A. Zadeh, University of California, Berkeley, for his inspirational address on the perceptual aspects of humans in the BISC seminars and group meetings.

\section{References}

[1] Choi, D. Y. (2006). Toward an intelligent local web search in telematics. LNAI 4251, 984-991.

[2] Choi, D. Y., \& Ra, I. K. (2010). Toward a voice interface and personalized local Web search in smartphones. RCIS 2010, 641-645.

[3] Google Mobile APP. From: http://www.google.com/mobile/google-mobile-app/

[4] Voice Control. From: http://www.apple.com/iphone/features/voice-control.html

[5] Franz, A., \& Milch, B. (2002). Searching the web by voice. Proceedings of 19th International Conference on Computational Linguistics, 1213-1217. 
[6] Kao, B., Lee, J., Ng, C. Y., \& Cheung, D. (2000). Anchor point indexing in web document retrieval. IEEE Trans. on SMC (Part C), 30(3), 364-373.

[7] Choi, D. Y. (2007). Personalized local internet in the location-based mobile Web search. Decision Support Systems, 43(1), 31-45.

[8] Choi, D. Y. (2002). Integration of document index with perception index. Soft Computing, 6(5), 300-307.

[9] Zadeh, L. A. (Aug. 2001). The problem of deduction in an environment of imprecision, uncertainty, and partial truth. In M. Nikravesh, \& B. Azvine (Eds.), New Directions in Enhancing the Power of the Internet, UC Berkeley Electronics Research Laboratory, Memorandum No. UCB/ERL M01/28.

[10] Personalization. From: http://en.wikipedia.org/wiki/Personalization

[11] Personalization definition. From: http://searchcrm.techtarget.com/definition/personalization

[12] Fensel, D., \& Musen, M. A. (Mar./Apr. 2001). The semantic Web: A brain for humankind. IEEE Intelligent Systems, 24-25.

[13] Smyth, B. (Aug. 2007). A community-based approach to personalizing Web search. IEEE Computer, 42-50.

[14] Supports, accounts. From: http://www.google.com/support/accounts/bin/topic.py?hl=en \&topic $=14148$

[15] Belkin, N. J. (2000). Helping people find what they don't know. Communications of the ACM, 43(8), 58-61.

[16] Zadeh, L. A. (1983). A computational approach to fuzzy quantifiers in natural language. Comput. Math. Appl., 9, 149-184.

[17] Yager, R. R. (1991). On linguistic summaries of data, In G. Piatetsky-Shapiro and B. Frawley (Eds.), knowledge Discovery in Databases (pp. 347-363). MIT Press.

[18] The Verge.

From: http://www.theverge.com/2012/5/163024418/google-knowledge-graph-intelligent-search

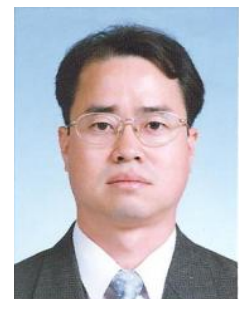

Dae Young Choi received the B.S., M.S., and Ph.D. degrees in computer sciences from Sogang University, Seoul, South Korea, in 1985, 1992, and 1996, respectively. He was a research fellow at the Korea Institute for Defense Analyses (KIDA), Seoul, from 1985 to 1990. He received a postdoctoral fellowship from the Korea Science and Engineering Foundation in 2000. He was with the Berkeley Initiative in Soft Computing (BISC) Group, Dept. of EECS, CS Division, Univ. of California, Berkeley, as a visiting scholar, in 2001. He was selected as a scholar to be sent abroad under the professor Dispatching Scheme by the Korea Research Foundation in 2004. He was with the Dept. of CSE, Univ. of Colorado at Denver as a visiting professor from 2004 to 2006. Dr. Choi was listed in Marquis Who's Who in Science and Engineering in 2003 and in Marquis Who's Who in the World from 2002 to2015). He is a Prof. in the Dept. of MIS, Yuhan Univ., Puchon, South Korea. He has a national certificate of Professional Engineer for information processing systems. He was an associate editor of the Transactions of the Korea Information Processing Society (Part B) and is on the editorial board of the International Journal of Information Processing Systems (IJIPS). His research interests include mobile Web search engines, personalized local Internet, business intelligence, fuzzy systems, and group decision support system (GDSS). 